\title{
Acesso a medicamentos no setor público: análise de usuários do Sistema Único de Saúde no Brasil
}

\author{
Access to medicines in the public sector: analysis \\ of users of the Brazilian Unified National \\ Health System
}

\author{
Acceso a medicamentos en el sector público: \\ análisis de usuarios del Sistema Único de \\ Salud en Brasil
}

\author{
Alexandra Crispim Boing 1 \\ Andréa Dâmaso Bertoldi 2 \\ Antonio Fernando Boing 1 \\ João Luiz Bastos 1 \\ Karen Glazer Peres 3
}

\section{Resumo}

This study aimed to describe the prevalence of access to medicines in the Brazilian Unified National Health System and associated factors among patients that received prescriptions in the public system. The study analyzed data from the National Household Sample Survey (2008). The sample consisted of individuals with prescriptions written in the National Health System in the two weeks prior to the interview $(n=19,427)$. The dependent variable was access to all the prescribed medicines inside the system itself. Slightly fewer than half of the sample with prescriptions received the medicines in the public health system; the proportions were higher in the South of Brazil, among black patients, those with less schooling and lower income, and those registered in the Family Health Strategy. When analyzing prescriptions written by the private sector, access to all the prescribed drugs was associated with higher income, higher education, and white skin color. The results emphasize the need for policies to decrease inequalities in access to medicines in Brazil.

Drug Utilization; Pharmacoepidemiology; Cross-Sectional Studies
Objetivou-se descrever a prevalência e os fatores associados ao acesso de usuários a medicamentos no Sistema Único de Saúde (SUS) que tiveram prescrição no próprio sistema público. Analisaram-se os dados da Pesquisa Nacional por Amostra de Domicílios conduzida em 2008, no Brasil. A amostra foi composta por indivíduos que tiveram medicamentos receitados no SUS nas duas semanas anteriores à entrevista $(n=19.427)$. A variável dependente foi o acesso à totalidade de remédios receitados no sistema. Pouco menos da metade da população que teve medicamentos prescritos os obteve no próprio sistema público, número mais elevado entre os habitantes da Região Sul, os de cor de pele preta, com menor escolaridade e renda e entre aqueles que residem em domicílios cadastrados na Estratégia Saúde da Família. Quando se considerou a obtenção desses fármacos via setor privado, verificou-se maior acesso total para os mais ricos, com maior escolaridade e brancos. Os resultados reforçam a necessidade de politicas que diminuam as desigualdades no acesso a medicamentos no país.

Uso de Medicamentos; Farmacoepidemiologia; Estudos Transversais 


\section{Introdução}

$\mathrm{O}$ acesso a medicamentos essenciais é reconhecido pela Organização das Nações Unidas como um dos cinco indicadores relacionados a avanços na garantia do direito à saúde 1. Estima-se que, no início do século XXI, uma em cada três pessoas no mundo não dispõe de acesso a esses insumos, sendo a pior situação verificada nos países de baixa e média renda, onde essa proporção pode chegar a $50 \% 2$. Nesses países, em apenas um terço das vezes, as instituições públicas têm os medicamentos essenciais disponíveis quando são procuradas pela população ${ }^{3}$. $\mathrm{O}$ acesso aos medicamentos se dá mediante a disponibilidade destes, a capacidade aquisitiva das pessoas, sua acessibilidade geográfica e aceitabilidade, levando ao uso racional do produto ${ }^{4}$. Estudos epidemiológicos demonstram que o acesso aos fármacos está associado com sexo feminino 5,6, maior idade 5,6,7, cor da pele parda e preta 5,8, nível socioeconômico mais elevado $5,7,8$, presença de doença crônicas 6,7,8 e número de consultas médicas realizadas 6,8 .

No Brasil, a disponibilização de medicamentos de forma contínua e em quantidade adequada às necessidades da população ainda é um desafio a ser superado, apesar da implementação de políticas públicas a partir da década de 1990, como a Política Nacional de Medicamentos (PNM), a Política Nacional de Assistência Farmacêutica (PNAF), a Política de Medicamentos Genéricos e o Programa Farmácia Popular ${ }^{9}$. Além disso, o gasto privado na aquisição de remédios é expressivo no país 10 , sobretudo ao se considerar a existência de um sistema de saúde público, com cobertura universal e sem pagamento direto dos usuários.

$\mathrm{O}$ acesso insuficiente aos medicamentos está diretamente associado com piora do estado de saúde, maior uso de terapias adicionais, aumento no número de retornos aos serviços de saúde e gastos adicionais nos tratamentos 6 . A existência de forte e inversa associação entre posição socioeconômica e subutilização de medicamentos evidencia que expressiva parcela da população tem o sistema público de saúde como única alternativa para viabilizar a terapêutica necessária 11 .

Quando considerados em conjunto o setor público e o privado, estudos conduzidos no Brasil evidenciam alta prevalência de acesso aos fármacos, porém com profundas desigualdades 6,7 . Prevalência mais elevada no acesso (da ordem de $90 \%$ ) foi verificada nos grupos populacionais com maior idade, renda e escolaridade; nos portadores de doença crônica; nos que participam de grupos de promoção de saúde nas unidades locais de saúde e naqueles cobertos pela Estratégia Saúde da Família (ESF) 7,12,13.

No entanto, os estudos de base populacional sobre acesso a medicamentos ainda são escassos no país $14,15,16$ e, frequentemente, restringem-se à oferta de serviços e medicamentos específicos 17 . Em adição, poucos trabalhos analisaram o acesso com distinção do setor em que se deu a prescrição e a aquisição (público ou privado) do remédio $6,7,13,18$.

Portanto, o objetivo deste estudo foi descrever a prevalência e os fatores associados ao acesso total, pelos usuários, a medicamentos no Sistema Único de Saúde (SUS) que tiveram prescrição no próprio sistema. Investigou-se, também, o meio de obtenção dos fármacos pelos indivíduos que não os receberam por intermédio do SUS, bem como a distribuição, segundo fatores socioeconômicos e demográficos, daqueles que não obtiveram, seja no setor público, seja no privado, quaisquer dos remédios prescritos.

\section{Métodos}

Foram analisados dados oriundos do suplemento saúde da Pesquisa Nacional por Amostra de Domicílios (PNAD). Conduzida em 2008 no Brasil pelo Instituto Brasileiro de Geografia e Estatística (IBGE), sua amostra $(n=391.868)$ foi representativa da população brasileira, inclusive a da área rural. Maiores detalhes do processo de amostragem da PNAD 2008 podem ser obtidos em documento oficial do IBGE 19.

A coleta de dados ocorreu nos domicílios das pessoas, mediante entrevistas face a face por equipe previamente treinada. As informações foram registradas em personal digital assistants (PDA) e os dados passavam simultaneamente por críticas e análise de consistência, por meio do Canadian Census Edit and Imputation System (CANCEIS). Em caso de ausência de um morador do domicílio ou crianças, o questionário foi respondido por outro membro do domicílio (entrevistas conduzidas por proxy).

No presente estudo, a população analisada correspondeu aos indivíduos que tiveram medicamentos receitados em atendimentos de saúde realizados no SUS, nas duas semanas anteriores à entrevista $(n=19.427)$. A variável dependente foi o acesso, pelo SUS, aos fármacos receitados em consultas realizadas no próprio sistema, ou seja, a obtenção de todos os medicamentos receitados no SUS. Esta informação foi obtida por meio da pergunta "Neste atendimento de saúde [realizado no SUS nas duas semanas anteriores à entrevista], recebeu gratuitamente os medicamentos receitados?". As respostas possíveis eram: 
(1) sim, todos os medicamentos; (2) sim, parte dos medicamentos e (3) não recebeu qualquer medicamento. As categorias (2) e (3) foram considerados como ausência de acesso total.

Como variáveis independentes exploratórias, foram analisadas macrorregião de residência no país (Norte, Nordeste, Centro-oeste, Sul e Sudeste), situação censitária de residência (urbana e rural), sexo (feminino e masculino), idade (0-9; 10-19; 20-29; 30-39; 40-49; 50-59; 60 anos ou mais); cor de pele/raça autorreferida (branca, preta e parda); anos de estudo (até 4; 5-8; 9-11 e 12 anos ou mais), renda per capita familiar em Reais (analisada em quintis), domicílio cadastrado na ESF (sim e não) e número de doenças crônicas autorreferidas (nenhuma; uma; duas ou mais). Foram investigadas doença de coluna ou costas, artrite ou reumatismo, câncer, diabetes, bronquite ou asma, hipertensão, doença do coração, insuficiência renal crônica, depressão, tuberculose, tendinite ou tenossinovite e cirrose. Os indivíduos que relataram cor de pele/ raça amarela [ $n=66$ ] e indígena [ $n=95$ ] foram excluídos da análise por representarem amostra reduzida.

Adicionalmente, foi analisado se as pessoas que não obtiveram os medicamentos via SUS realizaram sua compra (sim; não) e, dentre os que não compraram, qual foi o principal motivo (não tinha dinheiro; não encontrou o medicamento na farmácia; não havia farmácia próxima; recebeu o medicamento de um amigo, parente ou entidade filantrópica; tinha o medicamento em casa; não achou que todos eram necessários; começou a sentir-se melhor; ou outro motivo). Por fim, verificou-se a proporção de pessoas que não obtiveram quaisquer de seus medicamentos, seja no setor público, seja no privado, seja por doação, segundo as mesmas variáveis demográficas e socioeconômicas anteriormente descritas.

Todas as análises estatísticas foram realizadas no programa Stata 9.0 (Stata Corp., College Station, Estados Unidos), considerando-se o efeito de delineamento amostral e os pesos individuais. As análises bivariada e múltipla foram realizadas por meio da regressão logística ${ }^{20}$. A significância estatística do modelo e dos parâmetros referentes aos seus preditores foi verificada através do valor de $\mathrm{p}$ do teste de Wald. Foram incluídas no modelo múltiplo as variáveis exploratórias que apresentaram valor de $\mathrm{p}<0,20$ na análise bivariada e foram mantidas no modelo final aquelas com $\mathrm{p}<0,05$ no modelo múltiplo.

Para testar associação entre a proporção de pessoas que tiveram consulta de saúde no SUS nas duas semanas anteriores à entrevista sem obter quaisquer dos medicamentos prescritos e as variáveis demográficas e socioeconômicas, foi utilizado o teste de qui-quadrado de Rao-Scott 21 , o qual corrige o teste de proporções para o efeito de delineamento da amostragem complexa.

\section{Resultados}

A maior parte da amostra foi composta por mulheres $(59,4 \%)$, pessoas residentes na zona urbana $(82,7 \%)$, com idade entre 20 e 59 anos, que referiram cor/raça parda (50,5\%). Pouco mais de $60 \%$ reportaram ter até quatro anos de estudo e morar em domicílio cadastrado na ESF. Maiores detalhes da amostra estão descritos na Tabela 1.

Aproximadamente, duas em cada três pessoas que tiveram consulta no SUS nas duas semanas prévias à entrevista receberam prescrição de, ao menos, um medicamento. Nesse grupo, a prevalência de acesso a todos os fármacos no SUS foi de $45,3 \%$ (Tabela 1). Entre os que não os receberam por meio do SUS, $78,1 \%$ compraramnos no setor privado. Os principais motivos para a não aquisição dos medicamentos foram não ter dinheiro para comprá-los (51,5\%), não encontrar o medicamento na farmácia (14,6\%), ter recebido de um amigo ou por filantropia (6,9\%), ter os medicamentos em casa (5,8\%), achar que os medicamentos não eram necessários $(4,4 \%)$, começar a se sentir melhor (2,3\%); $12,7 \%$ responderam ser por outros motivos (Figura 1).

A Figura 2 apresenta a proporção de pessoas que não obtiveram, pelo SUS, pelo setor privado ou por doação, quaisquer dos medicamentos receitados. Observou-se gradiente negativo quanto à renda, sendo a não obtenção dos remédios aproximadamente duas vezes maior no quintil mais pobre. Pior perfil também foi observado entre aqueles com menor escolaridade $(p=0,029)$, quando comparados com os de maior escolaridade, e entre os pretos ( $\mathrm{p}=0,018)$, comparados aos brancos; jovens e adultos $(\mathrm{p}<0,001)$ também obtiveram menos medicamentos do que as crianças e os idosos, assim como os residentes do Norte ( $\mathrm{p}<0,001)$, quando comparados aos residentes do Sudeste do país. Ainda que os intervalos de confiança das variáveis escolaridade e cor da pele se sobreponham marginalmente, o teste de Rao-Scott, que corrige o efeito do delineamento, demonstra a existência de diferenças estatisticamente significativas.

Quando analisados, de modo exploratório, os fatores associados ao acesso total de medicamentos no SUS, as análises bivariadas indicaram que o odds de prevalência do desfecho foi maior entre os moradores da Região Sul do país (56\%), pretos e pardos (20\% e $12 \%$, respectivamente), entre os mais pobres (59\%), menos escolarizados (65\%) e com domicílio cadastrado na ESF (24\%). 
Tabela 1

Descrição da amostra e prevalência de acesso aos medicamentos no setor público, segundo variáveis sociodemográficas, de saúde e cadastramento na Estratégia Saúde da Família. Pesquisa Nacional por Amostra de Domicílios, Brasil, 2008.

\begin{tabular}{|c|c|c|c|c|}
\hline \multirow[t]{2}{*}{ Variável } & \multicolumn{2}{|c|}{ Amostra } & \multicolumn{2}{|c|}{ Prevalência de acesso aos medicamentos } \\
\hline & $\mathrm{n}$ & $\%$ & $\%$ & IC95\% \\
\hline \multicolumn{5}{|c|}{ Região de residência } \\
\hline Norte & 2.386 & 12,3 & 37,2 & $33,6-40,8$ \\
\hline Nordeste & 6.143 & 31,6 & 45,5 & $43,2-47,9$ \\
\hline Sudeste & 5.755 & 29,6 & 46,4 & $44,6-48,2$ \\
\hline Sul & 3.098 & 16,0 & 48,1 & $45,6-50,5$ \\
\hline Centro-oeste & 2.045 & 10,5 & 40,5 & $37,0-43,9$ \\
\hline \multicolumn{5}{|l|}{ Situação censitária } \\
\hline Rural & 3.357 & 17,3 & 46,5 & $43,5-49,5$ \\
\hline Urbana & 16.070 & 82,7 & 45,1 & $44,0-46,2$ \\
\hline \multicolumn{5}{|l|}{ Sexo } \\
\hline Masculino & 7.877 & 40,6 & 45,5 & $44,0-47,0$ \\
\hline Feminino & 11.550 & 59,4 & 45,2 & $43,9-46,5$ \\
\hline \multicolumn{5}{|l|}{ Idade (anos) } \\
\hline $0-9$ & 3.867 & 19,9 & 44,7 & $42,6-46,9$ \\
\hline $10-19$ & 2.127 & 11,0 & 47,7 & $45,0-50,4$ \\
\hline $20-39$ & 4.748 & 24,4 & 43,9 & $42,3-45,7$ \\
\hline $40-59$ & 5.206 & 26,8 & 46,4 & $44,7-48,2$ \\
\hline 60 ou mais & 3.479 & 17,9 & 44,7 & $42,8-46,6$ \\
\hline \multicolumn{5}{|l|}{ Raça/Cor } \\
\hline Branca & 7.965 & 41,3 & 43,6 & $42,1-45,1$ \\
\hline Preta & 1.579 & 8,2 & 48,1 & $45,2-51,1$ \\
\hline Parda & 9.722 & 50,5 & 46,4 & $44,9-47,8$ \\
\hline \multicolumn{5}{|c|}{ Escolaridade (anos) } \\
\hline 12 ou mais & 560 & 2,9 & 34,6 & $30,2-39,1$ \\
\hline $9-11$ & 3.093 & 16,0 & 41,2 & $39,2-43,2$ \\
\hline $5-8$ & 3.974 & 20,5 & 45,9 & $44,0-47,8$ \\
\hline Até 4 & 11.730 & 60,6 & 46,7 & $45,3-48,1$ \\
\hline \multicolumn{5}{|c|}{ Renda familiar per capita em quintis (em $\mathrm{R} \$$ ) } \\
\hline $1(0-125)$ & 3.831 & 20,1 & 50,8 & $48,6-53,0$ \\
\hline $2(126-209)$ & 3.784 & 19,9 & 46,6 & $44,4-48,7$ \\
\hline $3(210-328)$ & 3.812 & 20,0 & 47,2 & $44,2-49,3$ \\
\hline $4(329-485)$ & 3.801 & 20,0 & 43,4 & $41,4-45,4$ \\
\hline $5(485-6.628)$ & 3.797 & 20,0 & 39,4 & $37,5-41,2$ \\
\hline \multicolumn{5}{|c|}{ Domicílio cadastrado na ESF } \\
\hline Não & 7.121 & 36,8 & 42,0 & $40,4-43,7$ \\
\hline Sim & 12.224 & 63,2 & 47,3 & $45,9-48,7$ \\
\hline \multicolumn{5}{|l|}{ Doença crônica } \\
\hline Nenhuma & 8.434 & 43,4 & 44,9 & $43,4-46,5$ \\
\hline 1 & 4.711 & 24,3 & 47,2 & $45,3-49,0$ \\
\hline 2 ou mais & 6.282 & 32,3 & 44,6 & $43,0-46,1$ \\
\hline Total & 19.427 & & 45,3 & $44,2-46,5$ \\
\hline
\end{tabular}

ESF: Estratégia Saúde da Família; IC95\%: intervalo de 95\% de confiança. 
Figura 1

Fluxograma do acesso a medicamentos pelos usuários que tiveram consultas e prescrição de medicamentos no Sistema Único de Saúde, nas duas semanas anteriores à entrevista. Pesquisa Nacional por Amostra de Domicílios, Brasil, 2008.

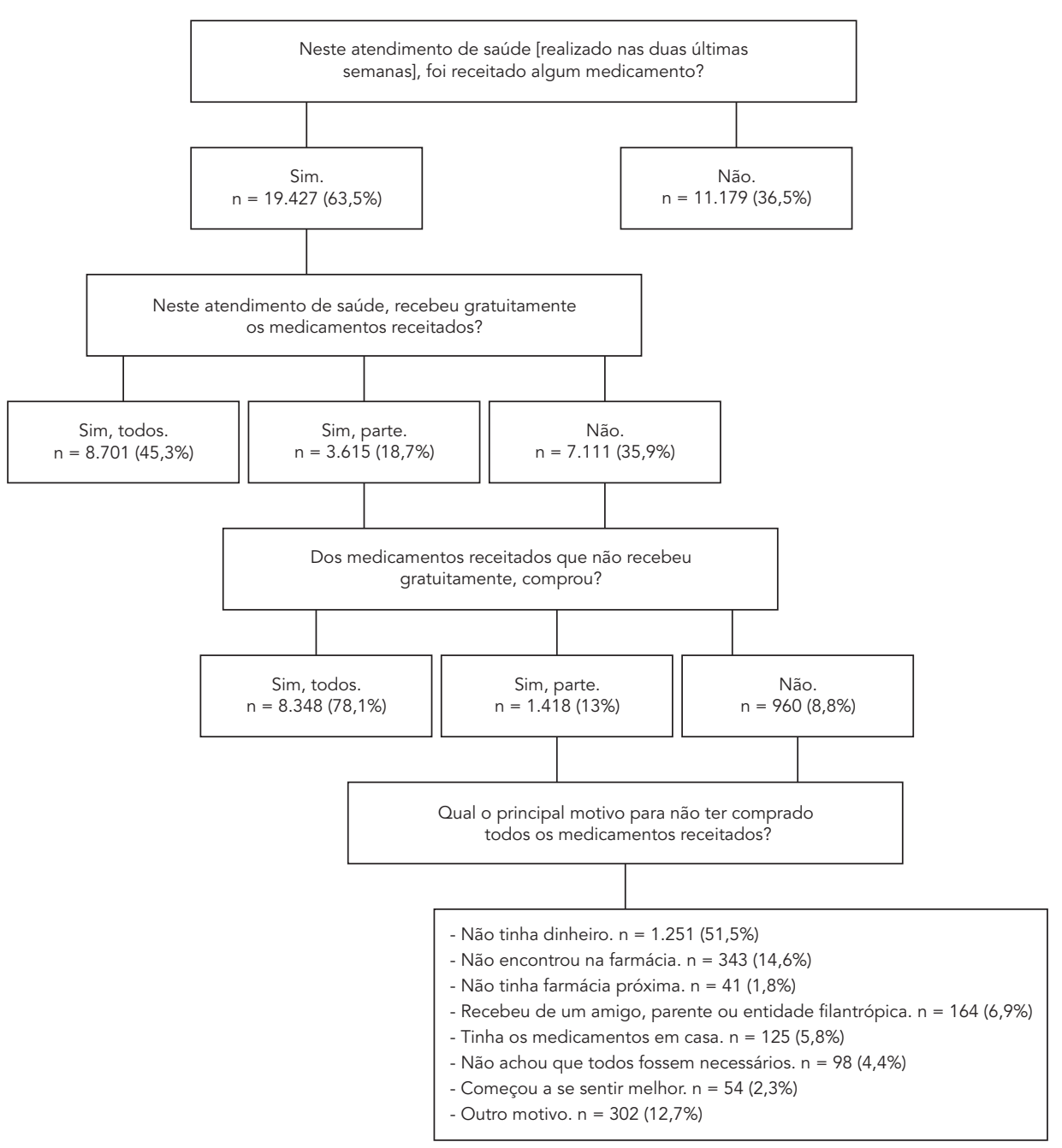

Na análise ajustada, todas as variáveis com associação significativa na análise bruta mantiveram a significância estatística e apresentaram diferenças na magnitude. O odds de prevalência de acesso total foi $85 \%$ maior entre os entrevistados da Região Sul, quando comparados aos da Região Norte; $21 \%$ e $16 \%$ maior entre os pretos e pardos em relação aos brancos; $36 \%$ maior entre as pessoas com quatro ou menos anos de estudo e $61 \%$ maior entre os $20 \%$ mais pobres, quando comparados com aqueles do quintil de maior renda (Tabela 2).

\section{Discussão}

O presente estudo identificou que pouco menos da metade da população que teve medicamentos prescritos no SUS os obteve no próprio sistema público; esse número foi mais elevado entre os habitantes da Região Sul, os de cor de pele preta, com menor escolaridade e renda e entre aqueles que residem em domicílios cadastrados na ESF. No entanto, quando se considerou também a obtenção desses remédios via setor privado, verificou-se maior acesso total para os mais ricos, com maior escolaridade e brancos. 
Figura 2

Proporção de pessoas que tiveram consulta de saúde no Sistema Único de Saúde nas duas semanas anteriores à entrevista e que não obtiveram quaisquer medicamentos prescritos, segundo variáveis sociodemográficas. Pesquisa Nacional por Amostra de Domicílios, Brasil, 2008.

2a) Quintil de renda

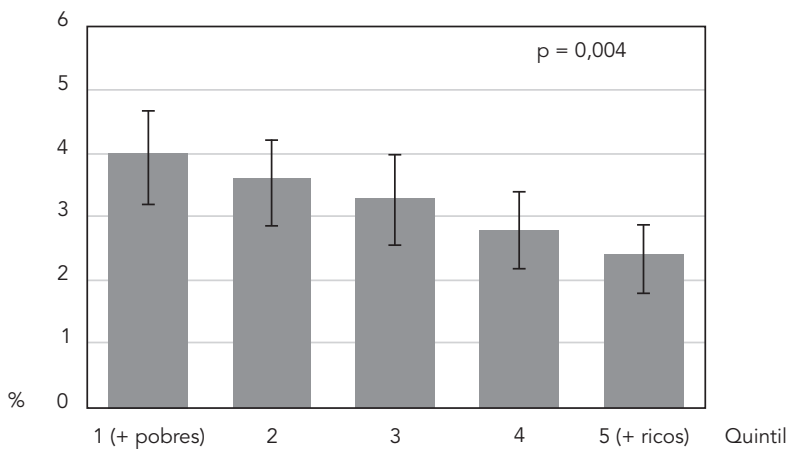

2c) Cor da pele

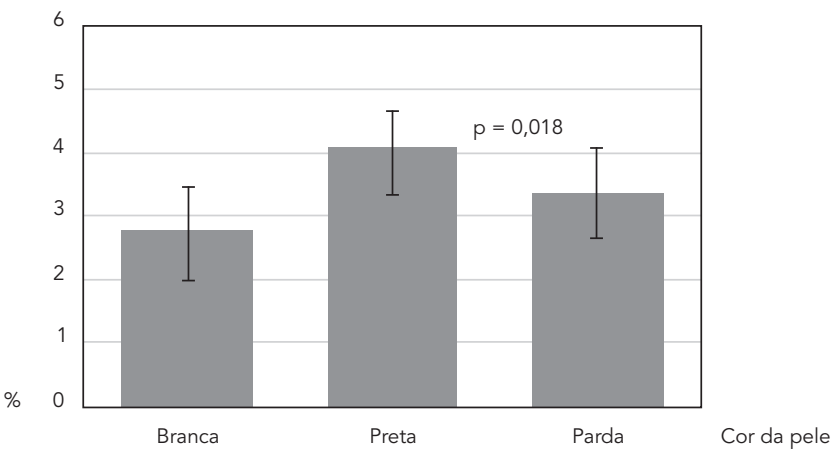

2e) Situação censitária

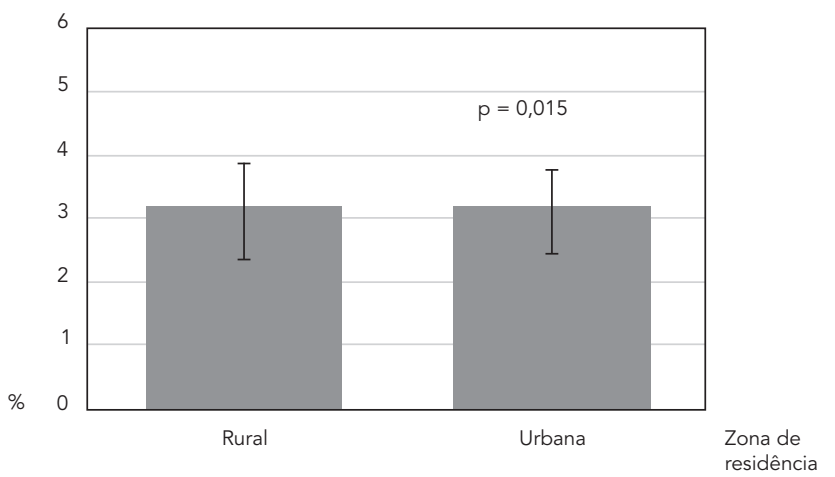

2b) Escolaridade

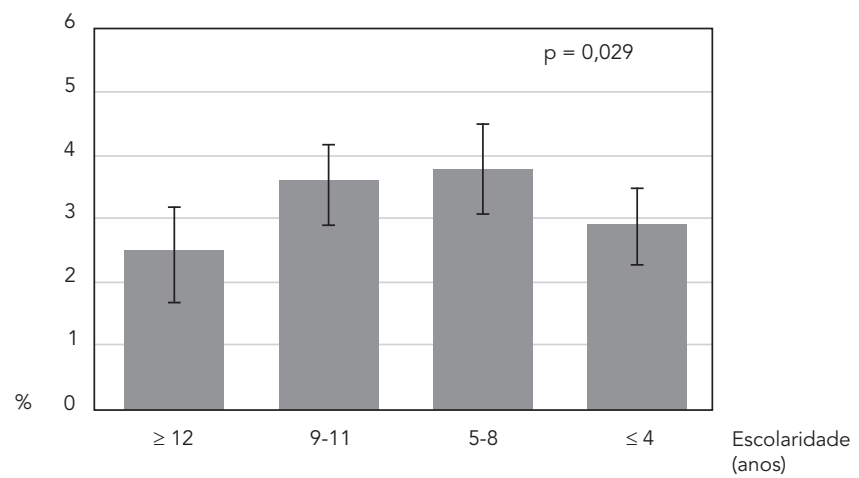

2d) Região de residência

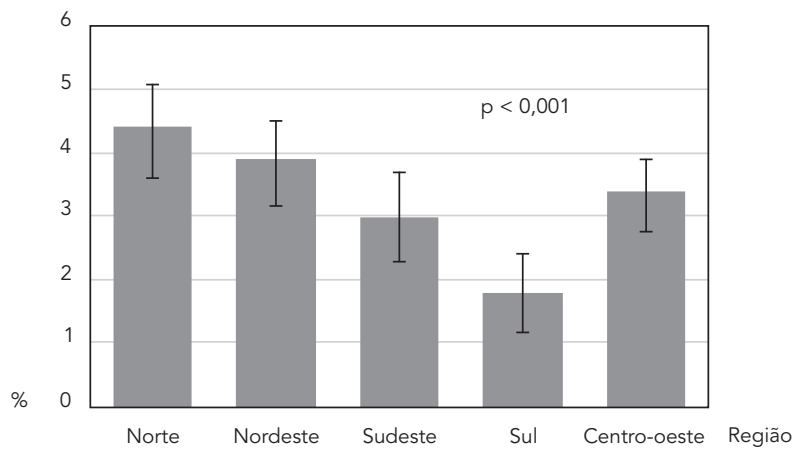

2f) Faixa etária

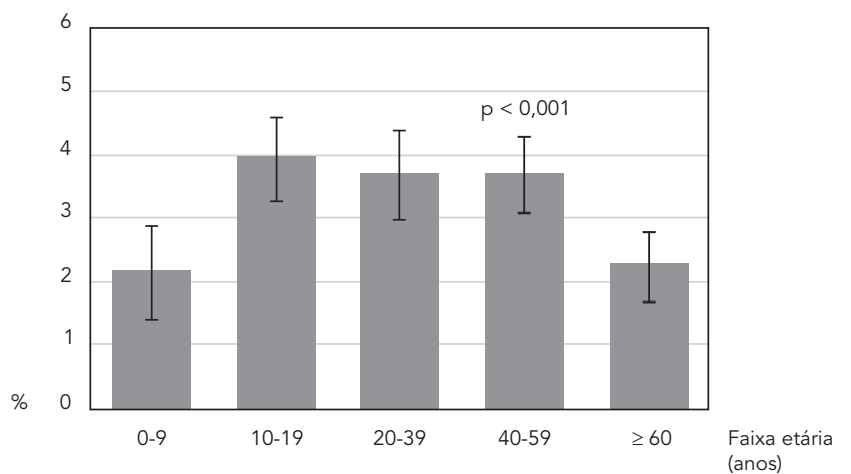


Tabela 2

Análise bruta e ajustada entre acesso a medicamentos dos usuários que tiveram consultas e prescrição de medicamentos nas duas semanas anteriores à entrevista no Sistema Único de Saúde e variáveis exploratórias. Pesquisa Nacional por Amostra de Domicílios, Brasil, 2008.

\begin{tabular}{|c|c|c|c|c|}
\hline Variáveis & OR bruta $(I C 95 \%)$ & Valor de $p$ * & $\mathrm{OR}_{\text {ajustada }}$ (IC95\%) & Valor de $p$ * \\
\hline Região de residência & & 0,076 & & $<0,001$ \\
\hline Norte & 1,00 & & 1,00 & \\
\hline Nordeste & $1,41(1,18-1,69)$ & & $1,32(1,13-1,62)$ & \\
\hline Sudeste & $1,46(1,24-1,72)$ & & $1,69(1,42-2,00)$ & \\
\hline Sul & $1,56(1,30-1,87)$ & & $1,85(1,53-2,23)$ & \\
\hline Centro-oeste & $1,15(0,93-1,41)$ & & $1,23(0,99-1,52)$ & \\
\hline Situação censitária & & 0,353 & ** & \\
\hline Rural & 1,00 & & & \\
\hline Urbana & $1,06(0,94-1,19)$ & & & \\
\hline Sexo & & 0,699 & ** & \\
\hline Masculino & 1,00 & & & \\
\hline Feminino & $1,01(0,95-1,08)$ & & & \\
\hline Idade (anos) & & 0,962 & $\star \star$ & \\
\hline $0-9$ & 1,00 & & & \\
\hline $10-19$ & $1,13(0,99-1,28)$ & & & \\
\hline $20-39$ & $0,97(0,88-1,07)$ & & & \\
\hline $40-59$ & $1,07(0,97-1,18)$ & & & \\
\hline 60 ou mais & $1,00(0,90-1,11)$ & & & \\
\hline Raça/Cor & & $<0,001$ & & 0,002 \\
\hline Branca & 1,00 & & 1,00 & \\
\hline Preta & $1,20(1,05-1,37)$ & & $1,21(1,06-1,39)$ & \\
\hline Parda & $1,12(1,04-1,20)$ & & $1,16(1,07-1,25)$ & \\
\hline Escolaridade (anos) & & $<0,001$ & & $<0,001$ \\
\hline 12 ou mais & 1,00 & & 1,00 & \\
\hline $9-11$ & $1,32(1,07-1,63)$ & & $1,15(0,92-1,44)$ & \\
\hline $5-8$ & $1,60(1,30-1,97)$ & & $1,34(1,08-1,67)$ & \\
\hline Até 4 & $1,65(1,35-2,02)$ & & $1,36(1,10-1,68)$ & \\
\hline $\begin{array}{l}\text { Renda familiar per capita em } \\
\text { quintis (em R\$) }\end{array}$ & \multicolumn{3}{|c|}{ quintis (em $\mathrm{R} \$$ ) } & $<0,001$ \\
\hline $5(485-6.628)$ & 1,00 & & 1,00 & \\
\hline $4(329-485)$ & $1,18(1,06-1,32)$ & & $1,16(1,04-1,29)$ & \\
\hline $3(210-328)$ & $1,38(1,25-1,53)$ & & $1,38(1,24-1,53)$ & \\
\hline $2(126-209)$ & $1,34(1,20-1,50)$ & & $1,33(1,19-1,49)$ & \\
\hline $1(0-125)$ & $1,59(1,42-1,79)$ & & $1,61(1,43-1,81)$ & \\
\hline Domicílio cadastrado na ESF & & $<0,001$ & & $<0,001$ \\
\hline Não & 1,00 & & 1,00 & \\
\hline Sim & $1,24(1,14-1,34)$ & & $1,21(1,11-1,31)$ & \\
\hline \multicolumn{5}{|l|}{ Doença crônica } \\
\hline Nenhuma & 1,00 & 0,816 & ** & \\
\hline 1 & $1,09(1,00-1,19)$ & & & \\
\hline 2 ou mais & $0,98(0,91-1,06)$ & & & \\
\hline
\end{tabular}

ESF: Estratégia Saúde da Família; IC95\%: intervalo de 95\% de confiança.

* Teste de Wald;

** Excluído da análise ajustada por apresentar $p \geq 0,20$ na análise bivariada. 
A prevalência de acesso aos medicamentos encontrada neste estudo foi de 45,3\%. Na etapa brasileira da Pesquisa Mundial de Saúde (PMS), em 2003, a prevalência de acesso total foi de $87 \%$ 12. Por sua vez, Paniz et al. 7, em 2005, ao analisarem populações do Sul e Nordeste do Brasil, encontraram que $81,2 \%$ dos adultos tiveram acesso total aos medicamentos de uso contínuo receitados; entretanto, é necessário cautela ao se compararem esses dados. Enquanto o presente estudo analisou prescrição e acesso apenas dentro do SUS, os demais incorporaram também fármacos prescritos e obtidos de outras fontes. Além disso, em virtude das diferenças entre as faixas etárias, tipos de medicamentos e períodos recordatórios investigados, comparações diretas devem ser feitas com ressalva 22.

O maior acesso evidenciado entre os de cor de pele preta, com menor escolaridade e menor renda não é comum na literatura 5,8 , contudo esse resultado pode ser decorrente de os estudos trabalharem com acesso aos medicamentos obtidos de qualquer fonte, independentemente de terem sido receitados e dispensados dentro do setor público. Dessa forma, esses grupos podem ter tido maior acesso por dependerem majoritariamente do SUS, ou seja, o sistema público é a principal alternativa para viabilizar o tratamento medicamentoso. Essa hipótese é fortalecida ao se observar que, ao mesmo tempo, esses grupos com maior acesso aos remédios no SUS têm maior dificuldade na obtenção dos insumos fora do sistema público, indicando que essas pessoas apresentam menor potencial de compra e maior dependência do setor público.

Tal achado reforça a suposição de que o SUS está promovendo a equidade no acesso aos medicamentos, contrapondo diferenças evitáveis e injustas entre grupos socioeconômicos e demográficos nesse acesso. Quando considerado o setor privado, as diferenças no acesso a esses insumos provavelmente seriam acirradas na ausência da dispensação gratuita de medicamentos no setor público, com prejuízo para os grupos desprivilegiados. Nesse sentido, Souza 23 também relatou o potencial do SUS em promover equidade no acesso aos medicamentos, ao analisar o fornecimento de anti-hipertensivos na cidade de São Paulo. Assim, sem comprometer seu caráter universal e igualitário, o SUS pode se configurar numa política pública de promoção de equidade 24 . Considerando-se que, em sociedades mais igualitárias, as pessoas tendem a ser mais saudáveis, tais políticas e ações podem seguir o conceito de universalismo proporcional, conforme proposto por Marmot Review Team 25, segundo o qual as ações devem ser universais e não focalizadas, com escalas e intensidade proporcional à desvantagem.
Apesar da relevância do SUS na provisão de fármacos, observou-se que apenas $45,3 \%$ das pessoas que têm medicamentos prescritos em consultas no sistema público os obtêm integralmente no próprio SUS. De fato, importantes avanços foram obtidos com a Política Nacional de Medicamentos e a Política Nacional de Assistência Farmacêutica, que estabeleceram definições e diretrizes para garantir o acesso aos medicamentos. Tais políticas definiram a utilização da lista de medicamentos essenciais (RENAME), a regulamentação do medicamento genérico, controle de preços, estímulo à produção dos laboratórios oficiais e reorientação da assistência com formação e capacitação de recursos humanos 26 .

Todavia, apesar das conquistas legais, maiores avanços dependem da seleção e uso racional de medicamentos, financiamento sustentado, preços acessíveis e adequado sistema de suprimento 27 , o qual ainda não está consolidado no Brasil. Para que se efetive o acesso aos fármacos pela população, é necessário que os governos melhorem sua capacidade de financiamento, uso eficiente dos recursos e regulação do mercado 28 .

Guerra et al. 29 analisaram a disponibilidade de medicamentos essenciais em municípios mineiros com Índice de Desenvolvimento Humano (IDH) menor que 0,699. Foi observado que a disponibilidade foi expressivamente maior nos estabelecimentos privados $(81,2 \%)$, em comparação com as unidades públicas de saúde $(46,9 \%)$. Ao analisarem estabelecimentos públicos, privados e do Programa Farmácia Popular no Brasil, Pinto et al. 15 também observaram no setor público os menores percentuais de disponibilidade total de fármacos, resultado também encontrado em cidades do Sul do país 16,30. Isto pode explicar o fato de que mais da metade das pessoas analisadas no presente estudo tiveram de recorrer a outros meios, fora do setor público, para viabilizar seu tratamento terapêutico. Destaca-se, por fim, que a migração dos usuários do SUS para o setor privado ou de copagamento para garantir o acesso aos medicamentos implica maior comprometimento de renda nos gastos em saúde, extremamente elevados ${ }^{31}$, que penalizam, sobretudo, os mais pobres 32 .

Em relação aos motivos para a não aquisição dos medicamentos, verificaram-se, no presente estudo, os mesmos da PMS em 2003 12. Segundo os dados da pesquisa, os principais motivos para que $13 \%$ das pessoas não adquirissem os remédios de que precisavam foram a impossibilidade de pagamento e a ausência do produto na farmácia. A falta de dinheiro foi determinante para a falta de acesso em estudo conduzido com 4.003 idosos, residentes em áreas cobertas por 
unidades básicas de saúde em 41 municípios do Sul e do Nordeste do país 33 .

No que diz respeito aos aspectos metodológicos, a amostra estudada neste artigo é representativa da população brasileira, permitindo a generalização dos resultados para toda a população. Entre as limitações do estudo, cabe destacar o potencial viés de memória envolvido nos dados autorreferidos, em particular no relato de realização de consultas no SUS e da forma de obtenção dos medicamentos prescritos. No entanto, destaca-se que o período recordatório foi curto (duas semanas), minimizando o problema.

$\mathrm{O}$ acesso aos medicamentos no país dentro do SUS ainda é insuficiente, mas o sistema público demonstra promover a equidade, sendo fundamental implementar estratégias para ampliar o acesso aos fármacos no país, de forma a diminuir as desigualdades no acesso a estes.

\section{Resumen}

Se tuvo como objetivo describir la prevalencia y los factores asociados al acceso de usuarios a medicamentos en el Sistema Único de Salud (SUS) y que recibieron prescripción en el propio sistema público. Se analizaron los datos de la Investigación Nacional por Muestreo de Domicilios, llevada a cabo en 2008, en Brasil. La muestra estaba compuesta por individuos a quienes les recetaron medicamentos en el SUS las dos semanas anteriores a la entrevista $(n=19.427)$. La variable dependiente fue el acceso a la totalidad de medicamentos recetados en el sistema. Poco menos de la mitad de la población que tenía medicamentos prescritos los obtuvo en el propio sistema público, el número más elevado fue entre los habitantes de la Región Sur, los afrobrasileños con menor escolaridad y renta, $y$ entre aquellos que residen en domicilios registrados en la Estrategia Salud de la Familia. Cuando se consideró la obtención de esos fármacos vía sector privado, se verificó un mayor acceso total para los más ricos, con mayor escolaridad y blancos. Los resultados refuerzan la necesidad de políticas que disminuyan las desigualdades en el acceso a medicamentos en el país.

Utilización de Medicamentos; Farmacoepidemiología; Estudios Transversales

\section{Colaboradores}

A. C. Boing, A. D. Bertoldi, A. F. Boing, J. L. Bastos e K. G. Peres contribuíram na concepção, planejamento, análise, interpretação dos dados, leitura crítica e aprovação da versão final do manuscrito. 


\section{Referências}

1. Hogerzeil HV, Mirza Z. The world medicines situation 2011: access to essential medicines as part of the right to health. Geneva: World Health Organization; 2011.

2. Organización Mundial de la Salud. Perspectivas políticas sobre medicamentos de la OMS. Geneva: Organización Mundial de la Salud; 2001.

3. Cameron A, Ewen M, Ross-Degnan D, Ball D, Laing R. Medicine prices, availability, and affordability in 36 developing and middle-income countries: a secondary analysis. Lancet 2009; 373:240-9.

4. World Health Organization; Management Sciences for Health. Defining and measuring access to essential drugs, vaccines, and health commodities. Report of Consultative Meeting. http://www.msh. org/seam (acessado em 10/Mai/2012).

5. Bertoldi AD, Barros AJD, Hallal PC, Lima RC. Utilização de medicamentos em adultos: prevalência e determinantes individuais. Rev Saúde Pública 2004; 38:228-38.

6. Arrais PSD, Brito LL, Barreto ML, Coelho HLL. Prevalência e fatores determinantes do consumo de medicamentos no Município de Fortaleza, Ceará, Brasil. Cad Saúde Pública 2005; 21:1737-46.

7. Paniz VMV, Fassa AG, Facchini LA, Bertoldi AD, Piccini RX, Tomasi E, et al. Acesso a medicamentos de uso contínuo em adultos e idosos nas regiões Sul e Nordeste do Brasil. Cad Saúde Pública 2008; 24:267-80.

8. Aziz MM, Calvo MC, Schneider IJC, Xavier AJ d'Orsi E. Prevalência e fatores associados ao acesso a medicamentos pela população idosa em uma capital do sul do Brasil: um estudo de base populacional. Cad Saúde Pública 2011; 27:1939-50.

9. Vieira FS. Assistência farmacêutica no sistema público de saúde no Brasil. Rev Panam Salud Pública 2010; 27:149-56.

10. Bertoldi AD, Barros AJD, Camargo AL, Hallal PC, Vandoros S, Wagner A, et al. Household expenditures for medicines and the role of free medicines in the Brazilian public health system. Am J Public Health 2001; 101:916-21.

11. Luz TCB, Loyola Filho AI, Lima-Costa MF. Estudo de base populacional da subutilização de medicamentos por motivos financeiros entre idosos na Região Metropolitana de Belo Horizonte, Minas Gerais, Brasil. Cad Saúde Pública 2009; 25:1578-86.

12. Carvalho MF, Pascom ARP, Souza-Júnior PRB, Damacena GN, Szwarcwald CL. Utilization of medicines by the Brazilian population, 2003. Cad Saúde Pública 2005; 21 Suppl 1:S100-8.

13. Bertoldi AD, Barros AJD,Wagner A, Ross-Degnan D, Hallal PC. Medicine access and utilization in a population covered by primary health care in Brazil. Health Policy 2009; 89:295-302.

14. Miranda ES, Pinto CDBS, Reis ALA, Emmerick ICM Campos MR, Luiza VL, et al. Disponibilidade no setor público e preços no setor privado: um perfil de medicamentos genéricos em diferentes regiões do Brasil. Cad Saúde Pública 2009; 25:2147-58.
15. Pinto CDBS, Miranda ES, Emmerick ICM, Costa NRO, Castro CGSO. Preços e disponibilidade de medicamentos no Programa Farmácia Popular do Brasil. Rev Saúde Pública 2010; 44:611-9.

16. Bertoldi AD, Helfer AP, Camargo AL, Tavares NUL, Kanavos P. Is the Brazilian pharmaceutical policy ensuring population access to essential medicines? Global Health 2012; 8:6.

17. Andrade, KVF, Sobrinho C, Lopes N, Silva MS, Rebouças BS. Indicadores do uso de medicamentos na atenção pediátrica em Feira de Santana. Rev Baiana Saúde Pública 2010; 34:333-47.

18. Loyola Filho AI, Uchoa E, Lima-Costa MF. Estudo epidemiológico de base populacional sobre uso de medicamentos entre idosos na Região Metropolitana de Belo Horizonte, Minas Gerais, Brasil. Cad Saúde Púbica 2006; 22:2657-67.

19. Instituto Brasileiro de Geografia e Estatística. Um panorama da saúde no Brasil. Acesso e utilização dos serviços, condições de saúde e fatores de risco e proteção à saúde 2008. Rio de Janeiro: Instituto Brasileiro de Geografia e Estatística; 2010.

20. Reichenheim ME, Coutinho ESF. Measures and models for causal inference in cross-sectional studies: arguments for the appropriateness of the prevalence odds ratio and related logistic regression. BMC Med Res Methodol 2010; 10:66.

21. Rao JNK, Scott AJ. The analysis of categorical data from complex sample surveys: chi-squared tests for goodness of fit the independence in two-way tables. J Am Stat Assoc 1981; 76:221-30.

22. Bertoldi AD, Barros AJD, Wagner A, Ross-Degnan D, Hallal PC. A descriptive review of the methodologies used in household surveys on medicine utilization. BMC Health Serv Res 2008; 8:222.

23. Souza JJG. Hipertensão arterial referida e uso de anti-hipertensivos em adultos na cidade de São Paulo, 2003: um estudo de base populacional [Dissertação de Mestrado]. São Paulo: Faculdade de Saúde Pública, Universidade de São Paulo; 2006.

24. Paim JS. Equidade e reforma em sistemas de serviços de saúde: o caso do SUS. Saúde Soc 2006; 15:34-46.

25. Marmot Review Team. Fair society, healthy lives: strategic review of health inequalities in England post-2010. Marmot Review 2010. http://www.mar motreview.org (acessado em Jun/2012).

26. Ministério da Saúde. Portaria no 399, 22 de fevereiro de 2006. Divulga o Pacto pela Saúde 2006. Consolidação do SUS e aprova as Diretrizes operacionais do Referendo Pacto. Diário Oficial da União 2006; 24 fev.

27. Organización Mundial de la Salud. Estrategia farmacéutica de la OMS: lo esencial son los países, 2004-2007. Geneva: Organización Mundial de la Salud; 2004.

28. Bermudez JAS, Oliveira MA, Escher A. Acesso a medicamentos: direito fundamental, papel do Estado. Rio de Janeiro: Escola Nacional de Saúde Pública Sergio Arouca, Fundação Oswaldo Cruz; 2004. 
29. Guerra Jr. AA, Acúrcio FA, Gomes CAP, Miralles M, Girardi SN, Werneck GAF. Disponibilidade de medicamentos essenciais em duas regiões de Minas Gerais, Brasil. Rev Panam Salud Pública 2004; 15:168-75.

30. Helfer AP, Camargo AL, Tavares NUL, Kanavos P, Bertoldi AD. Capacidade aquisitiva e disponibilidade de medicamentos para doenças crônicas no setor público. Rev Panam Salud Pública 2012; 31:225-32.

31. Silveira FG, Osório RG, Piola SF. Os gastos das famílias com saúde. Ciênc Saúde Coletiva 2002; 7:719-31.

32. Boing AC, Bertoldi AD, Peres KG. Desigualdades socioeconômicas nos gastos e comprometimento da renda com medicamentos no Sul do Brasil. Rev Saúde Pública 2011; 45:897-905.
33. Paniz VMV, Fassa AG, Facchini LA, Piccini RX, Tomasi E, Thumé E, et al. Free access to hypertension and diabetes medicines among the elderly: a reality yet to be constructed. Cad Saúde Pública 2010; 26:1163-74.

Recebido em 09/Jul/2012

Versão final reapresentada em 09/Out/2012

Aprovado em 29/Nov/2012 